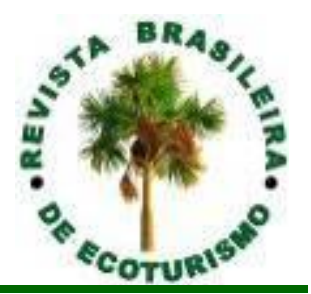

\title{
Fatores que influenciam a demanda por qualificação profissional para o desenvolvimento do ecoturismo no Brasil
}

\author{
Factors that influence the demand for professional qualification for the \\ development of ecotourism in Brazil
}

\author{
Cleber Augusto Trindade Castro, Patrícia Lins de Arroxelas Galvão, \\ Paula Wabner Binfaré
}

\begin{abstract}
RESUMO: Este trabalho trata do contexto da necessidade de oferta de qualificação profissional, especialmente em nível técnico, para o uso do potencial de desenvolvimento do ecoturismo no Brasil. Tal discussão torna-se relevante no contexto do paradigma da preservação de áreas naturais e da conservação de modos de vida ligados a essas áreas, a partir do conceito de desenvolvimento sustentável mundialmente difundido e que serve de pano de fundo para 0 desenvolvimento das ideias e princípios do ecoturismo, como estratégia de uso sustentável, empoderamento sociocultural e dinamização econômica para a preservação da natureza. O estudo está inserido no escopo da política nacional de expansão e interiorização da rede de educação profissional e tecnológica que ocorreu no Brasil na última década, abrangendo diferentes realidades socioambientais. Ainda assim, identifica-se a ausência da possibilidade de oferta de curso para a formação específica em ecoturismo pelo Eixo Tecnológico Turismo, Hospitalidade e Lazer, do Catálogo Nacional de Cursos Técnicos. Desse modo, objetivou-se identificar e analisar os fatores que influenciam a demanda por qualificação em nível técnico para a formação de profissionais para atuarem no desenvolvimento do ecoturismo no Brasil. O trabalho foi desenvolvido a partir do levantamento e análise documental em fontes de dados secundários a respeito do potencial do ecoturismo no Brasil e no mundo, bem como de dados sobre a política de proteção de áreas naturais no território brasileiro e sobre políticas públicas nacionais para o desenvolvimento do ecoturismo. O estudo aponta como principais fatores que subsidiam a necessidade da oferta de qualificação para o ecoturismo: a) o paradigma mundial do desenvolvimento sustentável, que embasa a adoção, pelo Estado brasileiro, de políticas nacionais de proteção de áreas naturais, exemplificadas pelo Sistema Nacional de Unidades de Conservação (lei $\mathrm{n}$ o $9.985 / 2000$, de 18 de julho de 2000), que tem produzido um mosaico de áreas naturais protegidas e estruturado o potencial ecoturístico do Brasil; b) a demanda mundial crescente pela prática do ecoturismo apontada por estudos feitos pela United Nations World Tourism Organization (2012), pelo Center for Responsible Tourism (2008) e pela International Ecotourism Society (2000); c) a existência de políticas públicas nacionais para o uso do potencial ecoturístico brasileiro, como é o caso do Programa de Desenvolvimento do Ecoturismo na Amazônia Legal (PROECOTUR), de 2000, além de diretrizes para o desenvolvimento do ecoturismo estarem presentes nos Planos Nacionais de Turismo, desde 2003.
\end{abstract}

PALAVRAS-CHAVE: Curso Técnico; Meio Ambiente; Áreas Protegidas; Desenvolvimento Sustentável; Ecoturismo. 


\section{ABSTRACT}

This paper deals with the context of the need for professional training offer, especially at the technical level, for the use of ecotourism development potential in Brazil. This discussion becomes relevant in the context of the paradigm of preserving natural areas and conservation lifestyles linked to these areas, from the concept of sustainable development worldwide widespread and serves as a backdrop for the development of ideas and principles ecotourism as a strategy for sustainable use, socio-cultural empowerment and economic dynamism to the preservation of nature. The study is inserted in the scope of national expansion and internalization policy of professional and technological education network that occurred in Brazil in the last decade, covering different environmental realities. Still, it identifies the absence of course offer the possibility for specific training in ecotourism for Technological Axis Tourism, Hospitality and Leisure, in the Technical Courses National Catalog. Thus, this study aimed to identify and analyze the factors that influence the demand for skills at a technical level for the training of professionals to work in the development of ecotourism in Brazil. The work was developed from document survey and analysis on secondary data sources regarding ecotourism potential in Brazil and worldwide, as well as data on the natural areas protection policy in Brazil and on national policies to ecotourism development. The study indicates that the main factors that support the need for qualifying offer for ecotourism: a) the global paradigm of sustainable development, that supports the adoption by the Brazilian state, national protection policies of natural areas, exemplified by the National System of Units of Conservation (law No. 9,985 / 2000 of 18 July 2000), which has produced a mosaic of protected natural areas and structured the ecotourism potential of Brazil; b) the global demand growing by ecotourism indicated by studies done by the United Nations World Tourism Organization (2012), the Center for Responsible Tourism (2008) and the International Ecotourism Society (2000); c) the existence of national policies for the use of Brazil's ecotourism potential, such as the Ecotourism Development Program in the Legal Amazon (PROECOTUR), 2000, as well as guidelines for the development of ecotourism are present in the National Plans Tourism since 2003.

KEYWORDS: Technical Course; Environment; Protected Areas; Sustainable Development; Ecotourism.

\section{Introdução}

As práticas de ecoturismo apresentam-se como alternativa e possibilidade de mitigação dos efeitos sociais, ambientais e econômicos desfavoráveis causados pelo desenvolvimento do turismo embasado na lógica de ganhos econômicos por qualquer via. Tais práticas atrelam-se à proposta de uma relação de integração dos turistas com o ambiente visitado, tendo como objetivo central a preservação dos elementos desse ambiente.

Diante desse contexto, desenvolve-se uma rede de profissionais e serviços para mediar as práticas e as experiências de ecoturismo, no âmbito mais amplo da mediação do turismo, de modo geral, o que também causa a demanda por qualificação de profissionais para atuarem a partir das especificidades técnicas que o desenvolvimento do ecoturismo exige e nas perspectivas da oferta de serviços com maior qualidade e de tornar o Brasil um país com destinos de ecoturismo mais competitivos. 
Assim, este artigo trata do contexto da necessidade de oferta de qualificação profissional, especialmente em nível técnico, para o uso do potencial de desenvolvimento do ecoturismo no Brasil, apresentando-se como uma contribuição às políticas educacionais, bem como às políticas de desenvolvimento do ecoturismo em território nacional.

Tal discussão torna-se relevante no contexto do paradigma da preservação de áreas naturais e da conservação de modos de vida ligados a essas áreas, a partir do conceito de desenvolvimento sustentável, mundialmente difundido e que serve de contexto para o desenvolvimento das ideias e princípios do ecoturismo, como estratégia de uso sustentável, empoderamento sociocultural e dinamização econômica para a preservação da natureza.

O estudo está inserido no escopo da política nacional de expansão e interiorização da Rede Federal de Educação Profissional, Científica e Tecnológica que ocorreu no Brasil nos últimos 13 anos, abrangendo diferentes realidades socioambientais. Ainda assim, identifica-se a ausência da possibilidade de oferta de curso para a formação específica em ecoturismo pelo Eixo Tecnológico Turismo, Hospitalidade e Lazer, do Catálogo Nacional de Cursos Técnicos.

As possibilidades de qualificação em nível técnico nesse eixo tecnológico, restritas à serviços que essencialmente abrangem realidades urbanas ou do turismo de modo geral, e a consequente lacuna da possibilidade de formação profissional específica ao ecoturismo, tornam esse trabalho uma contribuição e um subsídio ao debate acerca do acréscimo dessa possibilidade de qualificação.

Desse modo, objetivou-se identificar e analisar fatores que influenciam a demanda por qualificação em nível técnico para a formação de profissionais a atuarem no desenvolvimento do ecoturismo no Brasil, diante do potencial e das políticas que o país apresenta para essa estratégica.

O trabalho foi desenvolvido a partir do levantamento e análise documental em fontes de dados secundários a respeito do potencial do ecoturismo no Brasil e no mundo, bem como de dados sobre a política de proteção de áreas naturais no território brasileiro e sobre políticas públicas nacionais para o desenvolvimento do ecoturismo.

\section{Os paradigmas do desenvolvimento sustentável e o ecoturismo}

Os debates e as propostas que definem o ecoturismo apresentam relação com as discussões mais amplas travadas a partir dos anos de 1960 acerca das consequências socioambientais do desenvolvimento econômico e tecnológico manifestas até aquele momento.

Como produto dessas discussões, sobretudo na ocasião da Conferência das Nações Unidas sobre Meio Ambiente, realizada em 1972, em Estocolmo, é apresentado e difundido o conceito de desenvolvimento sustentável. Nesse conceito, está contida a ideia de que o crescimento econômico e os incrementos tecnológicos devem ser acompanhados da 
solução de problemas sociais históricos e da preservação dos elementos naturais que mantém o equilíbrio do planeta enquanto habitat, inclusive da vida humana, como está presente em United Nations (1987).

A partir da disseminação do conceito de desenvolvimento sustentável, os pressupostos trazidos por esse conceito passam a ter o caráter paradigmático, e a sustentabilidade passa a ser inserida em estudos para inovações nos modos de produção e nos outros âmbitos da dinâmica social.

Outro reflexo dessa disseminação é a ampliação da proteção de áreas naturais como estratégia de contenção do avanço da degradação ambiental causada por um desenvolvimento predatório. A partir do que Hosaka (2010) expõe, é possível notar que a década de 1970 foi o contexto da formação de uma rede de instituições transnacionais que estimularam a dotação de organizações nacionais para tratar de políticas ambientais, incluindo a proteção legal de áreas naturais.

O paradigma mundial do desenvolvimento sustentável, que embasa a adoção, pelo Estado brasileiro, de políticas nacionais de proteção de áreas naturais, exemplificadas pelo Sistema Nacional de Unidades de Conservação - SNUC (BRASIL, 2000), que tem produzido um mosaico de áreas naturais protegidas e estruturado o potencial ecoturístico do Brasil, como é demonstrado no mapa 1.

Mapa 1: Mosaico de áreas legalmente protegidas como Unidades de Conservação e Terras Indígenas, no território brasileiro.

Map 1: Mosaic of legally protected areas as conservation units and indigenous lands in Brazil.

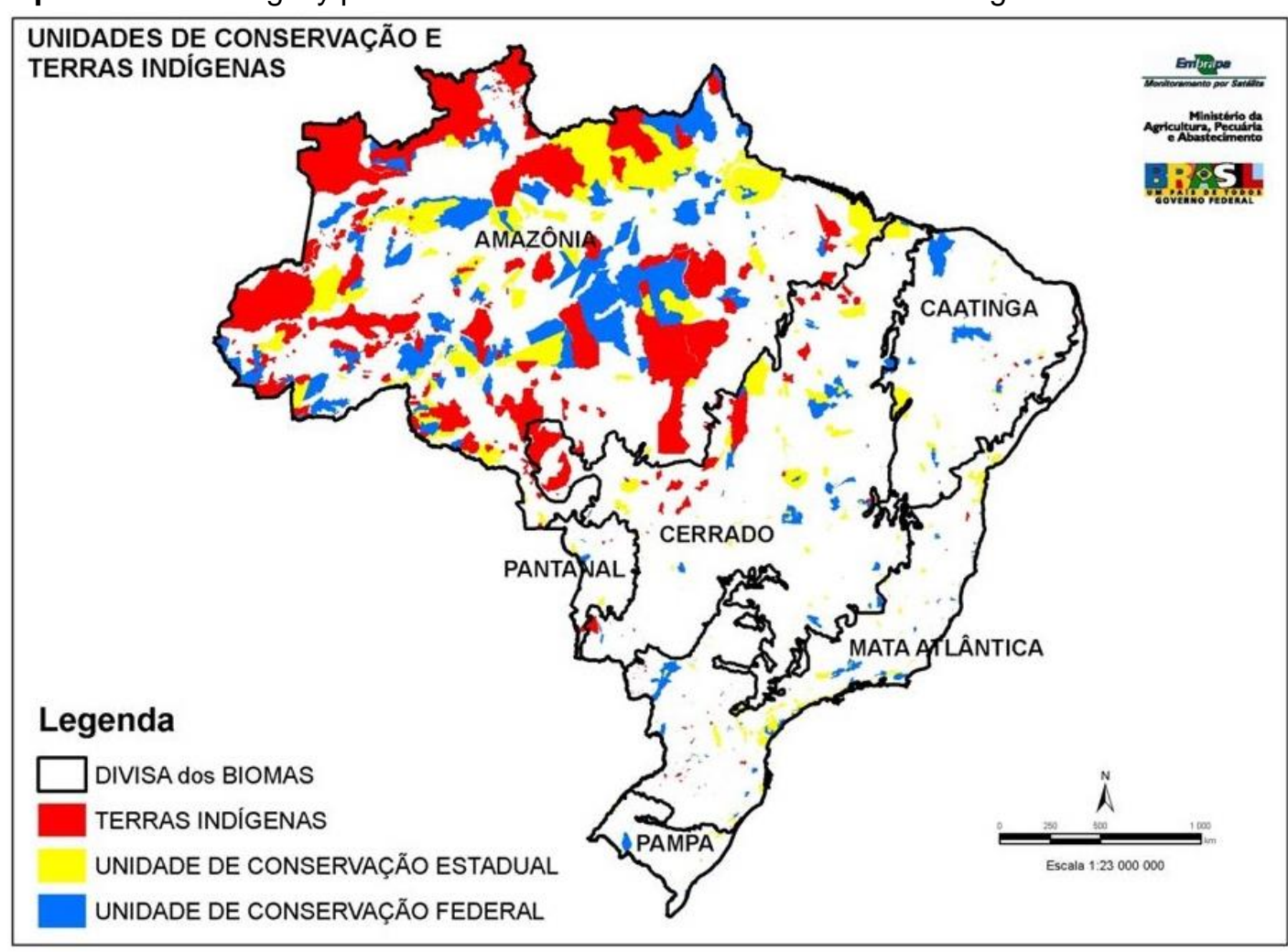

Fonte: EMBRAPA (2013).

Source: EMBRAPA (2013). 
Esse mosaico de áreas protegidas é o primeiro fator apontado nesse trabalho como um aspecto que contribui à demanda por qualificação profissional para atuar no desenvolvimento do ecoturismo no Brasil, pois, como indicam Wanderley Filha et al. (2013), o turismo é tomado como uma prática estratégica para 0 uso de algumas categorias de Unidade de Conservação e que para o planejamento e a implantação dessa visitação turística são necessários políticas específicas para as dinâmicas ambientais e territoriais dessas áreas.

Com as restrições de usos que provocam degradação a essas áreas naturais, são estimuladas práticas sociais e atividades produtivas que possibilitem a sustentabilidade, ou seja, a preservação ambiental, a dinamização econômica e o desenvolvimento social nessas áreas.

Nessa perspectiva, o ecoturismo, em sua concepção ampla, pode ser uma prática a estimular os usos sociais sustentáveis dessas áreas, a partir de aspectos teóricos e técnicos de planejamento e gestão do turismo específicos para esses objetivos, conforme indicam Chan e Bhatta (2013).

Ainda segundo esses autores, nessas especificidades incluem-se aspectos das dimensões sociais e culturais presentes nessas áreas, pois a desintegração entre ambiente e sociedade no planejamento do turismo em áreas protegidas tem se mostrada ineficiente para a promoção do desenvolvimento sustentável através do ecoturismo.

Como ampliação da noção de ecoturismo, comumente concebido apenas como prática turística em áreas naturais, ou como "turismo ecológico", Honey (1999) e Figueiredo (1999b) explicam que consiste na atividade turística que ao utilizar de maneira sustentável o patrimônio natural e cultural de um lugar, procura estimular a preservação desses elementos e promover a experiência de um contato que respeite os modos de vida e a dinâmica natural do destino ecoturístico.

Além disso, Melo, Maciel e Figueiredo (2015) e Figueiredo (1999a) exemplificam a intrínseca relação entre os elementos culturais e o ambiente natural para a eficiência da promoção turística de áreas naturais ainda preservadas. Portanto, o planejamento e a gestão de práticas de ecoturismo nas áreas naturais apresentam múltiplos aspectos técnicos que necessitam de qualificação profissional específica.

\section{Ecoturismo nas demandas de mercado e nas políticas para o turismo}

Desde o fim do período da Segunda Guerra Mundial, sobretudo a partir dos anos de 1950, o turismo está em crescente expansão no que diz respeito à quantidade de pessoas que o praticam e do volume de capital que movimentam em função dessa prática. Nesse contexto, também se expande a rede de organizações e profissionais que atuam na mediação das viagens e práticas turísticas (FIGUEIREDO, 2010).

Tal fato pode ser confirmado através das inúmeras pesquisas e publicações que apontam a atividade turística como um fenômeno possuidor de competências capazes de transformar vários aspectos de uma sociedade, entre eles a dinâmica econômica e as condições da vida sociocultural e o 
meio ambiente.

Dados do World Travel and Tourism Council mostram que, a atividade turística contribuiu, em 2011, com cerca de 10\% do total da dinâmica econômica mundial (2,9\% foi a contribuição direta) (WTTC, 2012). No Brasil, o turismo contribui com US $\$ 76.929,00$ de ganhos na economia (BRASIL, 2013).

Dados levantados pelo Center for Responsible Travel - CRT (2011) indicam que em 4 de cada 5 países (mais de 150) o turismo é um dos cinco maiores receptores de ganhos financeiros advindos de exportação, considerando os recursos que os turistas recebem em seu país de origem e gastam nos países visitados. Esses dados mostram os fluxos de capital e pessoas que turismo tem movimentado, o que é um potencial para 0 desenvolvimento humano dos destinos receptores desses fluxos.

Como a sociedade está em constante transformação, a atividade turística acompanha este movimento. Nos dias atuais, os turistas estão buscando cada vez mais viajar para lugares diferentes dos tradicionais, viajam em busca de novos destinos, e os países encontram neste "novo" modo de fazer turismo, o chamado "turismo alternativo", a oportunidade de mostrar o diferencial e a singularidade que possuem, seja pelos atrativos naturais, pelo patrimônio cultural, considerando a qualidade dos serviços.

Além disso, encontram-se também as possibilidades oferecidas pelas discussões dos movimentos ambientais presentes nas sociedades atuais que, consequentemente, influência nas diversas segmentações turísticas existentes que se enquadram nesta proposta ambiental como ecoturismo, agroturismo, turismo rural e etc.

Em conjunto com as reflexões acima mencionadas, vale destacar que a palavra diversidade revela muitas coisas sobre o Brasil. A política do Governo Federal de valorização da diversidade brasileira para incrementar 0 desenvolvimento do turismo brasileira em escalas nacional e internacional, em detrimento da imagem do país restrita a futebol e samba.

O país é diverso nos seus ambientes naturais, na sua cultura e nas raças que preenchem de valores únicos este vasto território. Diversidade é uma característica ímpar do Brasil e em face de tamanha vocação para o turismo, nada mais coerente do que "explorar", dentro dos princípios da sustentabilidade, todos os encantos que o país tem a oferecer.

Dentro deste novo momento do turismo no Brasil, a associação entre o Turismo e a Ecologia, tão em pauta atualmente, mostra-se uma excelente opção para a diversificação da oferta de produtos turísticos do país.

Segundo o CRT (2011), o ecoturismo tem captado US\$ 77 bilhões do mercado global e experimentando ganhos de dois dígitos que são susceptíveis de acelerar a partir do aumento da preocupação com o aquecimento global. A Organização Mundial de Viagens (OMT) estima que em 2007 o ecoturismo capturado 7 por cento do mercado internacional.

No Brasil, o ecoturismo é pauta de uma política pública específica desde a elaboração do Programa de Desenvolvimento do Ecoturismo na 
Amazônia Legal (PROECOTUR), de 2000 e vem sendo indicado nas diretrizes para o desenvolvimento do turismo dos Planos Nacionais de Turismo (BRASIL 2003; 2007; 2011).

Esse, portanto, é outro fator de demanda por qualificação de profissionais para atuar no ecoturismo como vetor de desenvolvimento do turismo no Brasil, dado os aspectos peculiares que a prática dessa atividade exige para o alcance desse objetivo.

\section{Educação Profissional e Tecnológica no Brasil}

As discussões estabelecidas nesse trabalho tornam-se relevantes, no contexto da expansão e interiorização da Rede Federal de Educação Profissional, Científica e Tecnológica, que passou a ampliar as possibilidades formação de força de trabalho para atuar em setores que igualmente visavam uma expansão.

Historicamente a formação de força de trabalho em nível técnico apresenta-se como uma estratégia de dotação de condições ao desenvolvimento de nações, sobretudo no que diz respeito aos setores industriais. No entanto, as mudanças socioeconômicas ocorridas a partir do fim da Segunda Guerra Mundial expandiram essa estratégia para outros setores produtivos, como a prestação de serviços e a produção artística e cultural, por exemplo.

Assim, a expansão quantitativa da oferta de qualificação técnica no Brasil está pautada nesse ajuste paradigmático, além de considerar as demandas crescentes por serviços de lazer e de hospitalidade e o potencial de contribuição ao desenvolvimento nacional que o turismo apresenta, apontado por Binfaré et al. (2016), pois o eixo tecnológico de Turismo, Hospitalidade e Lazer está presente naquele processo de expansão.

Nesse contexto, o Quadro 1 apresenta os dados quantitativos que contextualizam esse crescimento de oferta de campi dos Institutos Federais de Educação, Ciência e Tecnologia no Brasil, como contribuição ao desenvolvimento socioeconômico do país, por meio da preparação de trabalhadores para atuar em setores produtivos considerados estratégicos.

Quadro 1: Campi de instituições federais de ensino técnico e tecnológico no Brasil e municípios brasileiros atendidos

Table 1: Campi of federal institutions of technical and technological education in Brazil and served municipalities

\begin{tabular}{lllll}
\hline Período & $\begin{array}{l}\text { Campi } \\
\text { pré-existentes }\end{array}$ & $\begin{array}{l}\text { Campi } \\
\text { criados }\end{array}$ & $\begin{array}{l}\text { Total de } \\
\text { Campi }\end{array}$ & $\begin{array}{l}\text { Municípios } \\
\text { atendidos }\end{array}$ \\
\hline $1995-2002$ & - & - & 140 & 120 \\
$2003-2010$ & 140 & 214 & 354 & 321 \\
$2011-2014$ & 354 & 208 & 562 & 512 \\
\hline
\end{tabular}

Fonte: Elaborado com base em Brasil (2014). Source: Based in Brazil (2014). 
Entre outros aspectos, esses dados demonstram que o crescimento na quantidade de unidades institucionais para o ensino técnico, bem como o aumento do número de municípios e regiões atendidos por essas organizações, implicam na presença dessas instituições e das ações de qualificação em diferentes realidades sociais, culturais, ambientais e econômicas do Brasil.

A essa implicação está somada a presença de instituições de ensino próximos ou até mesmo na circunscrição de áreas naturais legalmente protegidas. Portanto, trata-se igualmente de um fator que pode ser considerado para a exigência de formação técnica específica em ecoturismo, considerando, como foi apresentado anteriormente, as motivações e consequências do uso turístico das Unidades de Conservação.

Todavia, quando analisamos o caso brasileiro das possibilidades legais de oferta de cursos técnicos na área de turismo, hospitalidade e lazer, observamos a impossibilidade da formação técnica especificamente na área de ecoturismo, como apresenta o quadro 2.

Quadro 2: Cursos técnicos do Eixo Tecnológico de Turismo, Hospitalidade e Lazer, no Catálogo Nacional de Cursos Técnicos.

Table 2: Technical courses of the Tourism, Hospitality and Leisure Technological Axis, in the National Technical Courses Catalog.

\begin{tabular}{ll}
\hline \multicolumn{1}{c}{ Eixo tecnológico } & \multicolumn{1}{c}{ Cursos } \\
\hline Técnico em Agenciamento de Viagem \\
Técnico em Cozinha \\
Técnico em Eventos \\
Turismo, Hospitalidade e Lazer & Técnico em Guia de Turismo em Hospedagem \\
Técnico em Lazer & Técnico em Restaurante Bar \\
\hline
\end{tabular}

Fonte: Brasil (2016).

Source: Brasil (2016)

Ao desconsiderar essa possibilidade, a política nacional de educação técnica também desatende realidades socioeconômicas específicas, em especial aquelas que habitam o mosaico de áreas naturais legalmente protegidas, em forma de Unidades de Conservação, apresentadas nesse trabalho.

Essa lacuna, portanto, corresponde a uma condição para o acréscimo da formação profissional de técnicos em ecoturismo, como perspectiva de ampliação qualitativa da política de qualificação de força de trabalho para atuar em setores estratégicos para o desenvolvimento do Brasil. 


\section{Considerações finais}

O estudo aponta como principais fatores que subsidiam a necessidade da oferta de qualificação para o ecoturismo: a) o paradigma mundial do desenvolvimento sustentável, que embasa a adoção, pelo Estado brasileiro, de políticas nacionais de proteção de áreas naturais, exemplificadas pelo Sistema Nacional de Unidades de Conservação, que tem produzido um mosaico de áreas naturais protegidas e estruturado o potencial ecoturístico do Brasil; b) a demanda mundial crescente pela prática do ecoturismo; c) a existência de políticas públicas nacionais para o uso do potencial ecoturístico brasileiro, como é o caso do PROECOTUR, além de diretrizes para o desenvolvimento do ecoturismo estarem presentes nos Planos Nacionais de Turismo, desde 2003.

Portanto, a inclusão da formação técnica em ecoturismo como possibilidade na política nacional de qualificação profissional tem amplo potencial de contribuição para o desenvolvimento socioeconômico nacional, sobretudo a partir da adoção da proteção de áreas naturais como estratégia de desenvolvimento sustentável.

\section{Referências}

BINFARÉ, P.; CASTRO, C.; SILVA, M.; GALVÃO, P.; COSTA, S. Planejamento turístico: aspectos teóricos e conceituais e suas relações com o conceito de turismo. Revista de Turismo Contemporâneo, Natal, v.4, Ed. Especial, abr 2016, pp. 24-40.

BRASIL. Catálogo Nacional de Cursos Técnicos. Brasília: Ministério da Educação, 2016.

BRASIL. Expansão da Educação Superior, Profissional e Tecnológica. Brasília: Ministério da Educação, 2014.

BRASIL. Mais turismo, mais desenvolvimento: indicadores. Brasília: Ministério do Turismo, 2013.

BRASIL. Plano Nacional de Turismo. Brasília: Ministério do Turismo, 2011.

BRASIL. Lei no 9.985/2000, de 18 de julho de 2000. Institui o Sistema Nacional de Unidades de Conservação da Natureza e dá outras providências. Brasília: Presidência da República, 2000.

BRASIL. Plano Nacional de Turismo. Brasília: Ministério do Turismo, 2007.

BRASIL. Plano Nacional de Turismo. Brasília: Ministério do Turismo, 2003.

CENTER FOR RESPONSIBLE TRAVEL. Responsible travel: global trends \& statistics. Washington: Stanford University, 2011.

CHAN, R.; BHATTA, K. Ecotourism planning and sustainable community development: theoretical perspectives for Nepal. South Asian Journal of Tourism and Heritage, v.6, n.1, jan 2013, pp. 1-56.

EMPRESA BRASILEIRA DE PESQUISA AGROPECUÁRIA. Alcance territorial das áreas protegidas pela legislação ambiental e indigenista. Brasília: EMBRAPA, 2013. 
FIGUEIREDO, S. Cultura e natureza: a viagem e o turismo como necessidades humanas. Revista de Turismo Contemporâneo, Natal, v.2, n.2, jul/dez 2014, pp. 283-299.

FIGUEIREDO, S. Viagens e Viajantes. São Paulo: Annablume, 2010.

FIGUEIREDO, S. Ecoturismo, festas e rituais na Amazônia. Belém: NAEA/UFPA, 1999a.

FIGUEIREDO, S. Ecoturismo e desenvolvimento sustentável: alternativa para o desenvolvimento da Amazônia? In: FIGUEIREDO, S. (Org.). O ecoturismo e a questão ambiental na Amazônia. Belém: NAEA/UFPA, 1999b, p. 75126.

HONEY, M. Ecotourism and sustainable development: Who owns paradise? Island Press, 1999.

HOSAKA, A. Unidades de conservação: aspectos históricos e conceituais. In: PHILIPPI JR.; RUSCHMANN, D (Eds.). Gestão ambiental e sustentabilidade no turismo. São Paulo: Manole, 2010.

MELO, J.; MACIEL, A.; FIGUEIREDO, S. Eventos culturais como estratégia de fomento do turismo: análise do Festival de Parintins (AM). Revista Brasileira de Ecoturismo, São Paulo, v.8, n.2, mai/ago 2015, pp. 251-272.

UNITED NATIONS. Our Common Future: Report of the World Commission on Environment and Development. Berna: World Commission on Environment and Development, 1987.

WANDERLEY FILHA, I.; AZEVEDO, F.; NÓBREGA, W.; ALBUQUERQUE, J. Planejamento e políticas públicas do turismo: uma discussão teórica no contexto das Unidades de Conservação do Brasil. Revista Brasileira de Ecoturismo, São Paulo, v.6, n.4, nov 2013, pp. 27-44.

WORLD TRAVEL AND TOURISM COUNCIL. Economic Impact Research. Disponível em: <www.wttc.org/research/>. Acesso em: 23/12/2012 
Cleber Augusto Trindade Castro: Universidade Federal Universidade Federal de Pernambuco, Recife, PE, Brasil.

E-mail: cleber.at.castro@gmail.com

Link para o currículo Lattes: http://lattes.cnpq.br/0347315623106403

Patrícia Lins de Arroxelas Galvão: Instituto Federal de Alagoas, Marechal Deodoro, AL, Brasil.

E-mail: parroxelas@yahoo.com

Link para o currículo Lattes: http://lattes.cnpq.br/7675452061314676

Paula Wabner Binfaré: Instituto Federal do Rio Grande do Norte, Natal, RN, Brasil.

E-mail: paulabinfare@gmail.com

Link para o currículo Lattes: http://lattes.cnpq.br/3784594579442577

Data de submissão: 13 de setembro de 2016

Data de recebimento de correções: 17 de outubro de 2018

Data do aceite: 17 de outubro de 2018

Avaliado anonimamente 